\title{
Quadratic forms over fields with finite square class number
}

by

\author{
K. SZYMnOzEK (Katowice)
}

Introduction. Throughout the paper, $k$ denotes a field of characteristic other than 2 and $g(k)=k^{*} / k^{* 2}$. We denote by $q$ the square class number of $k$, that is, $q=|g(k)|$.

In this paper we investigate the behaviour of the set of equivalence classes of quadratic forms over $k$ with respect to orthogonal sum of quadratic forms. This behaviour is characterized by the structure of Grothendieck group $G(7)$ of quadratic forms over $k$.

In Section 2 we describe completely the groups $G\left(k_{k}\right)$ for all fields with $q \leqslant 8$, by giving a decomposition of $G(k)$ into direct sum of cyclic groups and determining generators for the direct summands. It turns out that in the case of fields with $q=8$ there are at most 13 non-isomorphic groups $G(k), 7$ for non-real fields and 6 for real fields. A satisfactory feature of the classification is that the type of $G(k)$ is completely determined by the behaviour of binary quadratic forms over the field $k$ and by the reality of $\gamma$.

Another classification of Grothendieck groups has been supplied by W. Scharlat [12]. He classifies the groups $G(k)$ for fields with the propexty that the subgroup $B_{2}(k)$ of the Brauer group $B(k)$ consisting of elements of order $\leqslant 2$ has at most 4 elements. This class of fields contains even some fields with infinite square class number but it does not contain all the fields with $q=8$. In fact, if $Q(k)$ denotes the number of nonisomorphic quaternion algebras over $\hbar$, then clearly $Q(k) \leqslant\left|B_{2}(k)\right|$, so Scharlau's classification covers at most the fields with $Q \leqslant 4$. Now, as proved by $L$. Szczepanilk [15], in 5 of the 13 cases of fields with $q=8$ we have $Q>4$, hence those 5 cases do not fall under Scharlau's assumption (these are the cases IV and VII of Theorem 2.4 and IV, V, VI of Theorem 2.5 below). Moreover, Scharlau gives details only in the case $\left|B_{2}(k)\right|=2$ and for fields $k$ with $\left|B_{2}(k)\right|=4$ he gives only possible types of $G(k)$ modulo a subgroup of exponent 2, without specifying the properties of fields determining the type of $G(k)$. 
In Section 3 we obtain easily a parallel classification of Witt groups $W(k)$ of anisotropic quadratic forms over fields $k$ with $q \leqslant 8$. In the case of non-real fields with $q \leqslant 8$ a classification of Witt groups has been given in a recent paper of 0 . M. Cordes [1]. While the type of $W(k)$ in the present paper depends only on the behaviour of binary forms over $k$, Cordes uses the $u$-invariant and the number $Q$ of quaternion algebras to differentiate between the cases.

Let us describe briefly the contents of the two remaining sections. Section 1 contains a general study of sets of generators for the group $G(k)$, mostly without assuming that $q$ is tinite. In particular, in the case of a real field, we relate the structure of $G(7)$ to the number of orderings of $t /$ and investigate the influence of some extremal behaviour of binary forms over $k$ on the number of orderings of the field (Proposition 1.22) and on the structure of $G\left(F_{i}\right)$ (Propositions 1.11 and 1.23).

In Section 4 we collect a number of examples of fields with $q=8$ showing that all the cases, with the possible exception of two, of the classification theorems for Grothendieck groups actually do occur.

Notation. A diagonalized quadratic form $f=a_{1} x_{1}^{2}+\ldots+a_{n} x_{n}^{2}$, where $a_{i} \epsilon k^{*}$, will be denoted by $f=\left(a_{1}, \ldots, a_{n}\right)$ and its equivalence class by $\langle f\rangle=\left\langle a_{1}, \ldots, a_{n}\right\rangle$. For $a \epsilon k^{*}$ we shall use the bold-faced $a$ to denote the canonical image of $a$ in $g(k)$, i.e., $a=a k^{* 2}$. If a quadratic form $f$ represents an element $a$ in $k^{*}$, we write $f \approx a$. If $f \approx a$, then $f$ represents all the elements of $\boldsymbol{a}$; so we can speak of the representability of elements of $g(k)$ by a quadratic form. The subset of $g(k)$ represented by a form $f$ will be denoted $D(f)$. The number of elements of $g(k)$ represented by the form $(1,1)$. will be denoted by $q_{2}$. Thus $q_{2}=|D(1,1)|$. If $q_{2}=1$, the field is said to be pythagorean. A form $f$ is said to be universal if it represents all the elements of $k^{*}$ (or equivalently, if $D(f)=g(k)$ ).

If $k$ is non-real, we write $s=s(k)$ for the stufe of $k$. Thus $s$ is the minimal number of summands in a representation of -1 as a sum of squares. The stufe is always a power of two, as proved by A. Pfister in 1965 (cf. [11]]).

Infinite cyclic group will be denoted by $\boldsymbol{Z}$ (the group of rational integers) and a cyclic group of order $n$ by $\boldsymbol{Z} / n \boldsymbol{Z}$. A direct sum of $n$ copies of a group $G$ will be denoted by $G^{(n)}$. If $G$ is a group and $S$ is a subset of $G$, then we denote by $[S]$ the subgroup generated by $S$.

To avoid repetitions, let it be agreed that "field" will altways mean "field of characteristic other than 2 " and "form" will mean "a non-singular quadratic form".

1. Sets of generators for the Grothendieck group. We refer to [7], [10] and [13] for basic facts from the theory of quadratic forms over fields. Let $k$ be a field and $G(k)$ its Grothendieck group of quadratic forms.
In this section we do not assume that the square class number of $k$ is finite, unless otherwise stated. The following lemma is well known.

LEMMA 1.1. $G(k)=\boldsymbol{Z} \oplus G_{0}(k)$, where the infinite cyclie summand is generated by $\langle 1\rangle$ and $G_{0}(k)$, the subgroup of 0 -dimensional elements of $G\left(k_{i}\right)$, is generated by all the elements of the form $\langle 1\rangle-\langle c\rangle$, where $c$ runs through $k^{*}$.

Let us remark that in the caso when $g(k)$ is finite, the group $G(k)$ is a finitely generated abelian group and so, by a classical theorem, a direct sum of cyclic groups. It is also well known that the only possible torsion in $G(k)$ is at 2-power torsion.

LEMrua 1.2. If a is not a square in to and the form $(1, a)$ is universal and $g\langle k\rangle=\{\mathbf{1}, \boldsymbol{a}\} \times h$ (a direct product), then $G_{0}(k)$ is generated by $\langle 1\rangle-\langle a\rangle$ and all elements $\langle 1\rangle-\langle b\rangle$, where $\boldsymbol{b}$ runs through $h$.

Proof. $G_{0}$ is generated by all elements $\langle 1\rangle-\langle c\rangle, e \epsilon k^{*}$. Talke an element $\boldsymbol{a b}$ in $\boldsymbol{a} h$, then $\langle 1, a\rangle=\langle b, a b\rangle$. Hence $\langle 1\rangle-\langle a b\rangle=\langle 1\rangle-\langle a\rangle-$ $-(\langle 1\rangle-\langle b\rangle)$ and so $\langle 1\rangle-\langle a b\rangle$ may be omitted in the set of generators for $G_{0}$.

CoRoLIARX 1.3. If -1 is not a square in $k_{i}$ and $g\left(7_{0}\right)=\{\mathbf{1},-\mathbf{1}\} \times h$, then $G_{0}$ is generated by $\langle 1\rangle-\langle-1\rangle$ and all elements $\langle 1\rangle-\langle b\rangle$, where $\boldsymbol{b}$ runs through $h$.

LEMma 1.4. Let $g(k)=\prod_{i \in T}\left\{\mathbf{1}, \boldsymbol{a}_{i}\right\} \times h$, where $a_{i}$ are chosen in such a way that all the binary forms $\left(1, a_{i}\right), i \in I$, are universal. Then $G_{0}(7)$ is generated by all the elements $\langle 1\rangle-\left\langle a_{i}\right\rangle, i \in I$, and $\langle 1\rangle-\langle b\rangle$, where $b$ runs through $h$.

Proof. We shall prove that every element $\langle\mathbf{I}\rangle-\langle a b\rangle$, where $\boldsymbol{a} \epsilon \prod_{i \in I}\left\{\mathbf{I}, \boldsymbol{a}_{i}\right\}$ and $\boldsymbol{b} \in h$, can be represented as a linear combination with integer coefficients of a finite set of elements $\langle 1\rangle-\left\langle a_{i}\right\rangle$ and of $\langle 1\rangle-\langle b\rangle$. This follows from the following identity:

$$
\langle 1\rangle-\langle a b\rangle=\sum_{j=1}^{n}(-1)^{j+1}\left(\langle 1\rangle-\left\langle a_{j}\right\rangle\right)+(-1)^{n}(\langle 1\rangle-\langle b\rangle),
$$

where $a a_{1} \ldots a_{n}$. If $n=0$, then $a=1$ and the identity holds; if $n=1$, then $a=a_{1}$ and the result has been proved in Lemma 1.2. Assume the identity holds for sorne $n \geqslant 1$ and $a^{\prime}=a_{1} \ldots a_{n+1}=a_{1} a$, where $a=a_{2} \ldots$ $\ldots a_{n+1}$. Then

$$
\begin{aligned}
\langle 1\rangle-\left\langle a^{\prime} b\right\rangle & =\langle 1\rangle-\left\langle a_{1}(a b)\right\rangle=\langle 1\rangle-\left\langle a_{1}\right\rangle-(\langle 1\rangle-\langle a b\rangle) \\
& =\langle 1\rangle-\left\langle a_{1}\right\rangle-\left(\sum_{j=1}^{n}(-1)^{j+1}\left(\langle 1\rangle-\left\langle a_{j+1}\right\rangle\right)+(-1)^{n}(\langle 1\rangle-\langle b\rangle)\right) \\
& =\sum_{j=1}^{n+1}(-1)^{j+1}\left(\langle 1\rangle-\left\langle a_{j}\right\rangle\right)+(-1)^{n+1}(\langle 1\rangle-\langle b\rangle),
\end{aligned}
$$

as required. 
CoROLLARY 1.5. If the forms $\left(1, a_{i}\right), i=1, \ldots, n$, are universal and $a=a_{1} \ldots a_{n}$, then

$$
\langle 1\rangle-\langle a\rangle==\sum_{j=1}^{n}(-1)^{j+1}\left(\langle 1\rangle-\left\langle a_{j}\right\rangle\right) .
$$

LeimMA 1.6. Assume $g(k)=\prod_{i \in X}\left\{\mathbf{1}, \boldsymbol{a}_{i}\right\} \times h$, where $a_{i}$ is a sum of two squares, for all $i \in I$. Then the set of etements $\langle 1\rangle-\left\langle a_{i}\right\rangle, i \in I$, is linearly independent (over $\mathbf{Z}$ ).

Proof. First observe that all the elements $\langle 1\rangle-\left\langle a_{i}\right\rangle, i \in I$, are of order two since $\langle 1,1\rangle=\left\langle a_{i}, a_{i}\right\rangle$ for each $i \in I$. Assume now that

$$
\sum_{j=1}^{n}\left(\langle 1\rangle-\left\langle a_{i_{j}}\right\rangle\right)=0
$$

where $\left\{i_{1}, \ldots, i_{n}\right\}$ is a finite subset of $I$. Taking determinants on both sides we get

$$
\prod_{j=1}^{n} \boldsymbol{a}_{i_{j}}=\mathbf{1}
$$

a contradiction.

Now we are ready to determine the Grothendieck group in a case when the behaviour of binary forms makes the situation as simple as possible. But we assume nothing about the square class number of the field.

Propostrton 1.7 ([13], Theorem 4.1.1). Let $k$ be a field such that every binary form over $k$ is universal. Then

$$
\text { det: } G_{0}(k) \rightarrow g(k)
$$

is an isomorphism, and so $G(k) \cong \boldsymbol{Z} \oplus g(k)$.

Hence, if $\left\{\boldsymbol{a}_{i}: i \in I\right\}$ is any basis for $g(k)$, then $\left\{\langle 1\rangle-\left\langle a_{i}\right\rangle: i \in I\right\}$ is a basis for $G_{0}(7)$.

Proof. $G_{0}(k)$ is generated by all elements $\langle\mathcal{1}\rangle-\langle 0\rangle, c \in g(k)$. An application of Corollary 1.5 and Lemma 1.6 gives the result.

LaMma 1.8. Let $t$ be a field with square class number $2^{n}$. The number $u_{2}$ of universal binary classes over $k$ is a power of 2 and $u_{2} \leqslant 2^{n}$. If $h_{6}$ is non-real and $u_{2}<2^{n}$, then $u_{2} \leqslant 2^{n-2}$.

Proof. First observe that if the forms $(1, a)$ and $(1, b)$ are both universal, then so is $(1,-a b)$. This enables us to introduce the following law of composition in the set $U_{2}(k)$ of equivalence classes of binary universal forms:

$$
\langle 1, a\rangle \times\langle 1, b\rangle=\langle 1,-a b\rangle .
$$

This is clearly well defined on equivalence classes and makes $U_{2}(k)$ into an abelian group of exponent 2 with the identity $\langle 1,-1\rangle$. Hence $u_{2}$ $=\left|\sigma_{2}(k)\right|$, is a power of two and $u_{2} \leqslant 2^{n}$ (for details, see [18]). On the other hand, we denote by $R$ Kaplans.ky's radical of the field $k$, i.e. the set of those elements $\boldsymbol{a}$ in $g(7)$ which make any quaternion algebra $(a, b / k)$ split (cf. [6]). $R$ can be easily identified as the set of $a$ in $g(k)$ such that the class $\langle 1,-a\rangle$ is universal, and in fact the mapping sending $a$ in $R$ into $\langle 1,-a\rangle$ in $U_{2}(k)$ is an isomorphism. Hence $|R|=u_{2}$. Assume now that $u_{2}=2^{n-1}$. Then the index $\left[g\left(l_{0}\right): R\right]=2^{n} / 2^{n-1}=2$ and, by Lemma 2 of [6], $k$ is a real field with a unique ordering. Hence if $k$ is non-real, $u_{2} \neq 2^{n-1}$ and the lemma is proved.

Conoxitanx 1.9. If 7 is a non-real field and $q=4$, then either all binary forms over $k$ are universal or there exists exaotly one binary universal olass over $k$ (the hyperbolic plane). If $k$ is a non-real field and $q=8$, then either all binany forms over $k$ are universal or there are at most 2 universal binary classes over 7 .

We shall now turn to formally real fields and derive various structure theorems for Grothendieck groups of such fields. First we prove the following general lemma:

Liemma 1.10. Let $k$ be a real field and $g(k)=\{\mathbf{1},-\mathbf{1}\} \times h$, where all the elements of $h$ are positive in a fixed ordering of $k$. Then

$$
G(b) \cong Z \boldsymbol{Z} \oplus \boldsymbol{Z} \oplus G_{1}
$$

where the two infinite cyclic summands are generated by $\langle 1\rangle$ and $\langle\mathbf{1}\rangle-\langle-\mathbf{1}\rangle$, respectively, and $G_{1}$ is generated by all elements $\langle 1\rangle-\langle a\rangle, \boldsymbol{a} \epsilon h$.

Proof. In view of Lemma 1.1 it suffices to show that

$$
G_{0}(k)=[\langle 1\rangle-\langle-1\rangle] \oplus G_{1} .
$$

By Corollary 1.3 the group $G_{0}\langle k\rangle$ is generated by $\langle 1\rangle-\langle-1\rangle$ and all $\langle 1\rangle-\langle a\rangle, a \in h$. Hence it is sufficient to prove that from

$$
x_{0}(\langle 1\rangle-\langle-1\rangle)+\sum_{i=1}^{n} x_{i}\left(\langle 1\rangle-\left\langle a_{i}\right\rangle\right)=0,
$$

where $a_{i} \in h$, it follows $x_{0}=0$.

The above relation can be rewrititen as

$$
\left\langle b_{1}, \ldots, b_{m}\right\rangle=\left\langle c_{1}, \ldots, o_{n}\right\rangle,
$$

where $\left\{\boldsymbol{b}_{1}, \ldots, \boldsymbol{b}_{m}, \boldsymbol{c}_{\dot{x}}, \ldots, \boldsymbol{c}_{m}\right\} \subset\left\{\mathbf{1}, \boldsymbol{- 1}, \boldsymbol{a}_{1}, \ldots, \boldsymbol{a}_{n}\right\}$ and $\left\{\boldsymbol{b}_{1}, \ldots, \boldsymbol{b}_{m}\right\} \cap$ $\cap\left\{\boldsymbol{c}_{1}, \ldots, \boldsymbol{c}_{m}\right\}=\varnothing$.

If $x_{0} \neq 0$, then $-\mathbf{I}$ belongs to one of the sets $\left\{\boldsymbol{b}_{1}, \ldots, \boldsymbol{b}_{m}\right\}$ or $\left\{\boldsymbol{c}_{1}, \ldots\right.$ $\left.\ldots, c_{m}\right\}$; assume that $\boldsymbol{b}_{1}=-\mathbf{1}$. Then $\left\langle e_{1}, \ldots, c_{m}\right\rangle \approx-1$, a contradiction, 
since $c_{1}, \ldots, c_{m}$ are positive in the given ordering of the field. Hence $x_{0}=0$ and the lemma is proved.

We have already considered the case when every binary form over a field is universal. If $k$ is real, this cannot happen, but it is possible that "a half" of binary forms are universal (see the example 2.5. I in $\S 4$ below). We determine the group $G(k)$ for such a field $k$.

Propostrion 1.11. Let $k$ be a real field, $g(k)=\{\mathbf{1},-\mathbf{1}\} \times \prod_{i \in I}\left\{\mathbf{1}, \boldsymbol{a}_{i}\right\}$ and assume that all the binary forms $\left(1, a_{i}\right), i \in I$, are universal. Then

$$
G(k) \cong \boldsymbol{Z} \oplus \boldsymbol{Z} \oplus(\boldsymbol{Z} / 2 \boldsymbol{Z})^{[[]},
$$

where the two infinite cyclie summands are generated by $\langle 1\rangle$ and $\langle 1\rangle-\langle-1\rangle$ and the remaining summands are generated by the elements $\langle 1\rangle-\left\langle-a_{i}\right\rangle$, $i \in I$ :

Proof. By Lemma 1.4, $G_{0}(k)$ is generated by $\langle 1\rangle-\langle-1\rangle$ and all elements $\langle 1\rangle-\left\langle a_{i}\right\rangle, i \epsilon I$. Using the identity

$$
\langle 1\rangle-\langle a\rangle=\langle 1\rangle-\langle-1\rangle-(\langle 1\rangle-\langle-a\rangle)
$$

we can replace the above set of generators by $\left\{\langle 1\rangle-\langle-1\rangle,\langle 1\rangle-\left\langle-a_{i}\right\rangle\right.$ : $i \in I\}$. Now observe that $\langle 1\rangle-\langle-1\rangle$ is of infinite order and $2\left(\langle 1\rangle-\left\langle-a_{i}\right\rangle\right)$ $=0$, for all $i \in I$. Hence

$$
G_{0}=[\langle 1\rangle-\langle-1\rangle] \oplus G_{1}
$$

where $G_{1}$ is generated by the set $S=\left\{\langle 1\rangle-\left\langle-a_{i}\right\rangle: i \in I\right\}$. Since $\left(1, a_{i}\right)$ is universal, $-a_{i}$ is a sum of two squares; also $g(k)=\{\mathbf{1},-\mathbf{1}\} \times \prod\left\{\mathbf{1},-\boldsymbol{a}_{i}\right\}$, hence Lemma 1.6 applies and gives the result.

Our next goal is to relate the structure of the groups $g(k)$ and $G(k)$ to the number of orderings on the field $k$. If $a \in k$ is totally positive, then so is every element of $a=a k^{* 2}$. Hence we shall spealk of totally positive elements of the group $g(k)$. Obviously, they constitute a subgroup of $g(k)$. We have the following general result:

Proposition 1.12. Let $k$ be a real field and

$$
g(7)=\{\mathbf{1},-\mathbf{1}\} \times T \times \prod_{i \in I}\left\{\mathbf{I}, \boldsymbol{a}_{i}\right\}
$$

where $T$ denotes the subgroup of totally positive elements. of $g(7)$. The number $r=r(k)$ of ordorings of the field $\%$ is finite if and only if $I$ is finite. If $|I|=p<\infty$, then

$$
p+1 \leqslant r(k) \leqslant 2^{p}
$$

Proof. We assume first that $|I|=p<\infty$. In the case $p=0$, the set of totally positive elements defines an ordering of $k$ and hence the unique ordering. Thus $r=1$ and the theorem holds. Assume now $p \geqslant 1$. If

$$
P_{1}=T \times\left\{\mathbf{1}, \boldsymbol{a}_{1}\right\} \times \ldots \times\left\{\mathbf{1}, \boldsymbol{a}_{p}\right\}
$$

is an ordering of $k$ (we think here of $P_{x}$ as a subset of $k^{*}$.rather than of $g(k))$, then any other ordering of the field can be represented as

$$
P=T \times\left\{\mathbf{1}, \varepsilon_{\mathbf{1}} \boldsymbol{a}_{1}\right\} \times \ldots \times\left\{\mathbf{1}, \varepsilon_{p} \boldsymbol{a}_{p}\right\},
$$

where $\varepsilon_{j}= \pm 1$. Fence the number of orderings cannot exceed $2^{2 x}$. To prove the inequality $p+1 \leqslant r$, we represent all the $r$ orderings in the form

$$
P_{i}=T \times\left\{\mathbf{1}, \varepsilon_{i 1} \boldsymbol{a}_{\mathbf{1}}\right\} \times \ldots \times\left\{\mathbf{1}, \varepsilon_{i p} \boldsymbol{a}_{j p}\right\}, \quad i=1, \ldots, r,
$$

where $\varepsilon_{i j}= \pm 1$ and $\varepsilon_{1 j}=1, j=1, \ldots, p$.

Consider the matrix $M=\left[\varepsilon_{i j}\right], 1 \leqslant i \leqslant r, 1 \leqslant j \leqslant p$. The first row of $M$ consists of 1 's and any two rows are different. If a column, $j$ th say, consisted exclusively of 1 's, then $a_{j}$ would be positive at every ordering of $k$, hence $a_{j} \in T$, a contradiction. We denote by $E_{j}$ the $j$ th column of $M$ and define the componentwise multiplication of the columns. Observe that if a product $E_{j_{1}} \ldots D_{j_{t}}$ is equal to the unit vector (whose all components are 1), then

$$
a_{j_{1}} \ldots a_{j_{l}} \in P_{1} \cap \ldots \cap P_{r}=T,
$$

a contradiction. Consider the matrix $M$. The number of all possible products of columns one, two, etc, at a time, is at most

$$
\left(\begin{array}{l}
p \\
1
\end{array}\right)+\left(\begin{array}{l}
p \\
2
\end{array}\right)+\ldots+\left(\begin{array}{l}
p \\
p
\end{array}\right)=2^{p}-1
$$

If the number of distinct products was less than $2^{p}-1$, then we would have at least one equality of the type

$$
E_{j_{1}} \ldots E_{j_{t}}=E_{i_{1}} \ldots E_{i_{s}}, \quad\left\{j_{1}, \ldots, j_{t}\right\} \neq\left\{i_{1}, \ldots, i_{s}\right\}
$$

and this clearly produces a non-trivial product of the vectors equal to the unit vector. Fence the number of distinct vectors obtained by multiplying columns of the matrix $M$ is exactly $2^{p}-1$ and it cannot exceed $2^{r-1}$ (the total number of vectors with components $1, \pm 1, \ldots, \pm 1$ ). Hence $2^{p}-1 \leqslant 2^{r-1}$, and so $p \leqslant r-1$, if $p>1$. If $p=1$, then $a_{1}$ is not totally positive, hence $r \geqslant 2=p+r .1$.

To complete the proof, assume now that $r(k)<\infty$ and $|I|=\infty$. Let $p$ be any integer and $P_{i}=T \times \prod_{j \in T}\left\{\mathbf{1}, \varepsilon_{i j} a_{j}\right\}, i=1, \ldots, r$ be the $r$ orderings. Consider the first $p$ columns of the matrix [ $\varepsilon_{i j}$ ]. As above, any product of any number of these columns is different from the unit vector, hence $2^{p}-1 \leqslant 2^{r-1}$ for any integer $p$, a contradiction. Hence if $r$ is finite so is $I$, and the proposition is proved.

We recall that a field $k$ is said to be pythagorean if every sum of squares of elements of $k$ is a square itsolf. In a pythagorean field the subgroup $T$ of totally positive elements of $g(k)$ is trivial and we get the following result. 
CoRoLidary 1.13 (Elman and Lam [2], 4.5 and 5.7). Let to be a pythagorean field with $q=2^{n}$. Then $n \leqslant r \leqslant 2^{n-1}$.

A pythagorean field with $q=2^{n}$ and $r=2^{n-1}$ is said to be superpythagorean. The pythagorean fields with $q=2^{n}$ and $r=n$ can be shown to satisfy a certain strong approximation property (SAP, of: [2]). In the case $q=4$ both classes coincide; in the case $q=8$ the two classes of pythagorean fields are different.

CoRoutary 1.14. Let $k$ be a real field with a unique ordering. Then rank $G(k)=2$.

Pro of. If $r=1$, then $g(k)=\{\mathbf{1},-\mathbf{1}\} \times T$, where $T$ is thes sulbgromp of totally positive elements. By Lemma 1.10 we have $G\left(k_{i}\right) \approx \boldsymbol{Z} \oplus \boldsymbol{Z} \boldsymbol{Z} \oplus G_{1}$, where $G_{1}$ is generated by all elements $\langle 1\rangle-\langle a\rangle, a \in T$. By Artin-Schreiex theorem, any such $a$ is a sum of squares, hence a sum of $2^{m}$ squares, for an integer $m$, hence $2^{m}(\langle 1\rangle-\langle a\rangle)=0$. Thus $G_{1}$ is a torsion group and $\operatorname{rank} G(7)=2$

Now we want to establish that in the case $r=2$ we have rank $G(k)=3$. First we reformulate some well known Pfister's results concerning the Witt ring $W(k)$ for the case of Grothendieck ring $G(k)$. Let $k$ be a real field and $P$ an ordering of $k$. The map

$$
\sigma_{\mathcal{P}}: M(k) \rightarrow Z, \quad \sigma_{P}\left\langle a_{1}, \ldots, a_{n}\right\rangle=\sum_{i=1}^{n} \operatorname{sgn}_{P} a_{i}
$$

is a semiring homomorphism ( $M(7)$ is the semiring of equivalenco classes of non-singular quadratie forms over $k$ ), hence by the universal property of Grothendieck ring, $\sigma_{P}$ factors uniquely through a ring homomorphism $G(k) \rightarrow Z$, which will also be denoted by $\sigma_{p}$. Hence

$$
\sigma_{\mathcal{P}}(\langle f\rangle-\langle g\rangle)=\sigma_{P}\langle f\rangle-\sigma_{P}\langle g\rangle .
$$

Now using standard arguments one can easily deduce trom the results of [11] the following Pfister's Local-Global Principle:

(1.15) $A \in G(k)$ is torsion if and only if $\operatorname{dim} A \ldots 0$ and $\sigma_{x^{\prime}}(A) \pm 0$ at every ordering $P$ of the field th.

We denote by $k_{P}$ the real closure of $z_{0}$ which induces the ordering $P$ on $k$. Further, let $j_{P}: G\left(k_{i}\right) \rightarrow G\left(l_{p_{P}}\right)$ be the canonical mappring and $G^{l}\left(k_{k}\right)$ the torsion subgroup of $G(k)$. Then an equivalent formulation of $(1.15)$ is the following.

(1.16). The sequence

$$
0 \rightarrow G^{t}(k) \rightarrow G_{0}(k) \stackrel{M_{j_{p}}}{\longrightarrow} \prod G_{0}\left(k_{P}\right)
$$

is escact.
Now we can prove the following assertion.

Propostatow 1.17. Let to be a real field and $g(k)=\{\mathbf{1},-\mathbf{1}\} \times\{\mathbf{1}, \boldsymbol{a}\} \times T$, where $T$ is the subgroup of totally positive elements of $g\left(l_{c}\right)$. Then

$$
G(k)=Z^{(s)} \oplus G^{t}(k),
$$

where the three infinite cyclio summands are generated by $\langle 1\rangle,\langle 1\rangle-\langle-1\rangle$, $\langle 1\rangle--\langle a\rangle$, respeotively.

Proof. From Proposition 1.12 we obtain $r=2$. Let $P_{1}$ and $P_{2}$ be the two orderings of $k_{t}$ and $k_{1}$ and $k_{2}$ the two corresponding real closures of $\%$. Oonsider the mapping

$$
\left(j_{1}, j_{2}\right): G_{0}\left(k_{i}\right) \rightarrow G_{0}\left(k_{1}\right) \times G_{0}\left(k_{2}\right)
$$

Wo have

$$
\left(j_{1}, j_{2}\right)(\langle 1\rangle-\langle a\rangle)=(\langle 1\rangle-\langle-1\rangle, 0)
$$

and

$$
\left(j_{1}, j_{2}\right)(\langle 1\rangle-\langle-\infty\rangle)=(0,\langle 1\rangle-\langle-1\rangle),
$$

where we have assumed that $a \in P_{2}$ and $-a_{\epsilon} P_{1}$. This shows that the homomorphism. $\left(j_{1}, j_{2}\right)$ is surjective. Since the group $G_{0}\left(k_{1}\right) \times G_{0}\left(k_{2}\right)$ is free, we have

$$
G_{0}\left(T_{0}\right)=[\langle 1\rangle-\langle a\rangle] \oplus[\langle\mathcal{1}\rangle-\langle-a\rangle] \oplus \operatorname{Ker}\left(j_{1}, j_{2}\right)
$$

by a standard lemma on abelian groups (cf. [8], p. 44).

From (1.16) we obtain

$$
\operatorname{Ker}\left(j_{1}, j_{2}\right)=G^{t}\left(k_{i}\right),
$$

and the identity

$$
\langle 1\rangle-\langle a\rangle+\langle 1\rangle-\langle-a\rangle=\langle 1\rangle-\langle-1\rangle
$$

shows that

$$
[\langle 1\rangle-\langle a\rangle] \oplus[\langle 1\rangle-\langle-a\rangle]=[\langle 1\rangle-\langle-1\rangle] \oplus[\langle 1\rangle-\langle a\rangle],
$$

which yroves the proposition.

Remsurk 1.18. Using Whan and Lam's Normality Theorem [2] one can prove that for any field with finite number $r$ of orderings one has rank $G(k)=r+1$. This has been noticed by $A$. Sladek (for details and an independent elementary proof, see [14]]). However, the proof in the general case does not point out any basis for the group $G(k)$.

Now wo will prove two results concorning pythagorean fields.

Proposstion 1.19. A field $k$ is pythagorean if and only if the Grothendieoth group $G(7)$ is torsion free. 
Proof. If $k$ is not pythagorean, then $(1,1)$ represents a non-square $a$, say, and then $\langle 1,1\rangle=\langle a, a\rangle$ and $2(\langle 1\rangle-\langle a\rangle)=0$, i.e. $\langle 1\rangle-\langle a\rangle \neq 0$ is a torsion element in $G(k)$.

To prove the converse we need a lemma.

Leinca 1.20. Let 7 be a pythagorean field and $n$ a positive integer. Then for any positive integer $m$,

$n\left\langle a_{1}, \ldots, a_{m}\right\rangle=n\left\langle b_{1}, \ldots, b_{m}\right\rangle \Rightarrow\left\langle a_{1}, \ldots, a_{m}\right\rangle=\left\langle b_{1}, \ldots, b_{m}\right\rangle$.

Proof by induction on $m$. Consider first the case $m=1$. If $n\langle a\rangle=n\langle b\rangle$, then $n\langle a\rangle \approx b$. But $k$ is pythagorean, hence $\langle a\rangle \approx b$, and $\langle a\rangle=\langle b\rangle$

Assume the lemma holds for forms of dimension $m$.

If $n\left\langle a_{1}, \ldots, a_{m+1}\right\rangle=n\left\langle b_{1}, \ldots, b_{m+1}\right\rangle$, then $n\left\langle a_{1}, \ldots, a_{m+1}\right\rangle \approx b_{m+1}$ and by pythagoreanity, $\left\langle a_{1}, \ldots, a_{m+1}\right\rangle \approx b_{m+1}$.

Consequently $\left\langle a_{1}, \ldots, a_{m+1}\right\rangle=\left\langle c_{1}, \ldots, c_{m}, b_{m+1}\right\rangle$ for some $c_{1}, \ldots, c_{m}$. Now $n\left\langle 0_{1}, \ldots, c_{m}, b_{m+1}\right\rangle=n\left\langle b_{1}, \ldots, b_{m+1}\right\rangle$ and by Witt's cancellation theorem ([19], Satz 4) we get $n\left\langle a_{1}, \ldots, o_{m}\right\rangle=n\left\langle b_{1}, \ldots, b_{m}\right\rangle$. Applying the induction hypothesis we obtain $\left\langle o_{1}, \ldots, o_{m}\right\rangle=\left\langle b_{1}, \ldots, b_{m}\right\rangle$ and finally

$$
\left\langle a_{1}, \ldots, a_{m+1}\right\rangle=\left\langle e_{1}, \ldots, e_{m}, b_{m+1}\right\rangle=\left\langle b_{1}, \ldots, b_{m+1}\right\rangle,
$$

as required.

Now we may complete the proof of Proposition 1.19. Assume $k$ is pythagorean. If there is a torsion element $A$ in $G\left(l_{0}\right)$ we write $A=\left\langle f_{1}\right\rangle-\left\langle f_{2}\right\rangle$. and assume $n A=0, n\rangle^{\circ} 0$. Then $n\left\langle f_{1}\right\rangle=n\left\langle f_{2}\right\rangle$ and so $\operatorname{dim} f_{1}=\operatorname{dim} f_{2}$ and Lemma 1.20 applies: $\left\langle f_{1}\right\rangle=\left\langle f_{2}\right\rangle$, i.e. $A=0$. Thus there is no nontrivial torsion in $G(k)$.

PropostTION 1.21. Let $k$ be a pythagorean field and

$$
g(k)=\{\mathbf{1},-\mathbf{1}\} \times \prod_{i \in I}\left\{\mathbf{1}, \boldsymbol{a}_{i}\right\} .
$$

Then the set $\mathscr{B}=\left\{\langle 1\rangle-\langle-1\rangle ;\langle 1\rangle-\left\langle a_{i}\right\rangle: i \in I\right\}$ is linearly independent. In particular, if $q=|g(k)|=2^{n}$, then rank $G(k) \geqslant n+1$.

Proof. In this proof the symbol $(a, b)$ denotes the quaternion algebra corresponding to $a, b \in k^{*}$. We shall use the Hasse algebra and apart from its standard properties ([10], \& 58) we shall make use of the following straightforward consequence of the definition:

For positive integers $x_{i}$ one has

$$
S\left(\sum_{i=1}^{n} x_{i}\left\langle a_{i}\right\rangle\right) \sim \otimes_{i=1}^{n}\left(a_{i},\left(a_{1}^{x_{1}} \ldots a_{i-1}^{x_{i}}\right)^{x_{i}} a_{i}^{x_{i}\left(x_{i}+1\right) / 2}\right) .
$$

Assume now that a finite subset of $\mathscr{B}$ is linearly dependent. Then $x_{1}\left(\langle 1\rangle-\left\langle b_{1}\right\rangle\right)+\ldots+x_{r}\left(\langle 1\rangle-\left\langle b_{r}\right\rangle\right)$

$$
=y_{1}\left(\langle 1\rangle-\left\langle 0_{1}\right\rangle\right)+\ldots+y_{s}\left(\langle 1\rangle-\left\langle e_{s}\right\rangle\right)
$$

where all the coefficients are positive integers. Moreover, Proposition 1.19 makes it clear that we can assume the coefficients $x_{i}, y_{j}$ not to be all even. Multiplying by 2 and rearranging the summands we obtain

$$
\begin{aligned}
A & =2\left(x_{1}+\ldots+w_{r}\right)\langle 1\rangle+2 y_{1}\left\langle o_{1}\right\rangle+\ldots+2 y_{s}\left\langle o_{s}\right\rangle \\
& =2\left(y_{1}+\cdots+y_{s}\right)\langle 1\rangle+2 x_{1}\left\langle b_{1}\right\rangle+\ldots+2 x_{r}\left\langle b_{r}\right\rangle=B .
\end{aligned}
$$

These two quadratic forms have isomorphic Hasse algebras. But

$$
\begin{aligned}
S A & \sim S\left(2 y_{\perp}\left\langle c_{1}\right\rangle+\ldots+2 y_{s}\left\langle o_{s}\right\rangle\right) \\
& \sim \otimes_{i=1}^{s}\left(c_{i},\left(e_{1}^{2 y_{1}} \ldots c_{i-1}^{2 y_{i-1}}\right)^{2 y_{i}} c_{i}^{y_{i}\left(2 y_{i}+1\right)}\right) \\
& \sim \otimes_{i=1}^{\otimes}\left(c_{i}, c_{i}^{y_{i}}\right) \sim\left(c_{i_{1}},-1\right) \otimes \ldots \otimes\left(c_{i_{l}},-1\right) \\
& \sim\left(-1, c_{i_{1}} \ldots c_{i_{l}}\right)
\end{aligned}
$$

where $i_{1}, \ldots, i_{i}$ are those indices $i$ for which $y_{i}$ is odd. Similarly, $S B \sim$ $\sim\left(-1, b_{j_{1}}, \ldots, b_{j_{m}}\right)$, where $j_{1}, \ldots, j_{m}$ are those indices $j$ for which $x_{j}$ is odd. Thus $S A \sim S B$ implies $\left(-1, a_{i_{1}} \ldots c_{i_{l}}\right) \cong\left(-1, b_{j_{1}} \ldots b_{j_{m}}\right)$, hence $o_{i_{1}} \ldots o_{i_{l}} b_{j_{1}} \ldots b_{j_{m}}$ is a sum of two squares, hence a square, since the field is pythagorean.

But $\boldsymbol{c}_{i}, \boldsymbol{b}_{j}$ aro elements of a basis for $g(k)$, hence their product cannot be a scuare. This shows that the set $\mathscr{B}$ is linearly independent.

Wo end this section with some observations relating the number of orderings of a pythagorean field to the behaviour of binary forms over the field.

Proposircon 1.22. Let $k$ be a real field with square olass number $q=2^{n}$ $\geqslant 4$ and $r$ orderings.

(i) To is superpythagorean (i.e. $r=2^{n-1}$ ) if and only if $k$ is pythagorean and every anisotropio binary form represents at most 2 elements of $g(k)$.

(ii) If $t h$ is pythagorean and there exists an anisotropio binary form representing more than 2 elements of $g(7)$ and $n \geqslant 3$, then $r \leqslant 3 \cdot 2^{n-3}$.

Proof. Jet $\left\{-\mathbf{1}, \boldsymbol{a}_{1}, \ldots, \boldsymbol{a}_{n-1}\right\}$ be a basis for the group $g(7)$. As observed by Wlman and Lam ([2], j). 1181) two orderings on $7_{0}$ are the same if and only if aach $a_{i}$ has the same sign under both orderings. If $r=2^{n-1}$, then all possible combinations of signs do occur and so every element of $g(\%)$ differenti from 1 is negative at a certain ordering. Fence $D(1,1)=1$ and $z$ is pythagorean. If $q=4$ we cheek at once $\left|D\left(1, a_{1}\right)\right|=\left|D\left(1,-a_{1}\right)\right|$ $=2$ and this proves the assertion. Assume $q \geqslant 8$. Let $(a, b)$ be an anisotropic form, i.e. $\boldsymbol{a b} \neq \ldots \mathbf{1}$. If $\boldsymbol{a b}=\mathbf{1}$, then $D(a, b)=\boldsymbol{a} D(1,1)=\boldsymbol{a}$, i.e. $|D(a, b)| \leqslant 2$, as required. If $\boldsymbol{a b} \neq \mathbf{I}$, then $|D(a, b)|=|D(1, a b)|$ and so wo can consider the form $(1,0)$, where $c \neq \pm 1$. Fix an ordering $P$ 
at which $c$ is positive (this is possible for any pythagorean field by [2], Prop. 4.1). Assume $(1, c) \approx d$, where $\boldsymbol{d} \neq \mathbf{1}$ and $\boldsymbol{d} \neq \mathbf{c}$. Then also $\boldsymbol{d} \neq-\mathbf{1}$ and $\boldsymbol{d} \neq-\boldsymbol{c}$ since every element represented by $(1, c)$ is positive at $P$. Thus $\boldsymbol{d}$ does not belong to the subgroup of $g(k)$ generated by $-\mathbf{1}$, $\boldsymbol{c}$ and we can choose a basis for $g(k)$ of the form $\left\{-\mathbf{1}, \boldsymbol{c}, \boldsymbol{d}, \boldsymbol{b}_{3}, \ldots, \boldsymbol{b}_{n-1}\right\}$. Since $d$ is positive whenever $\theta$ is, we obtain $r \leqslant 3 \cdot 2^{n-3}$, which proves (ii). But if $r=2^{n-1}$ this is impossible, hence $|D(a, b)|=|D(1, c)|=2$ and the first part of (i) is proved. Conversely, assume that $k$ is pythagorean and $|D(a, b)|$ $\leqslant 2$ for $\boldsymbol{a} b \neq-\boldsymbol{1}$. Let $\left\{-\mathbf{l}, \boldsymbol{a}_{1}, \ldots, \boldsymbol{a}_{n-1}\right\}$ be any basis for $g(k)$. We prove that the subgroup $P$ of $g(k)$ generated by $\left\{\boldsymbol{a}_{1}, \ldots, \boldsymbol{a}_{n-1}\right\}$ defines an ordering of the field. Clearly, $P \cup-P=g(k)$ and $P$ is closed under multiplication. Take arbitrary $a$ in $\boldsymbol{a}$ and $\beta$ in $\boldsymbol{b}$, where $\boldsymbol{a}, \boldsymbol{b} \in P$. Then $\boldsymbol{a b} \neq-\mathbf{1}$ and $(\alpha, b) \approx \alpha+\beta$, hence $\alpha+\beta \epsilon \boldsymbol{a}$ or $\alpha+\beta \epsilon \boldsymbol{b}$. This shows that $P$ (as a subset of $k^{*}$ ) is closed under addition, hence $P$ defines an ordering of $k$. But if $\left\{-\mathbf{1}, \boldsymbol{a}_{1}, \ldots, \boldsymbol{a}_{n-1}\right\}$ is a basis for $g(k)$ then so is $\left\{-\mathbf{1}, \pm \boldsymbol{a}_{1}, \ldots, \pm \boldsymbol{a}_{n-1}\right\}$ for any choice of the signs; hence there are $2^{n-1}$ distinct orderings and the proposition is proved.

Proposmion 1.23. If $k$ is a superpythagorean field with square olass number $q=2^{n}$, then

$$
G(k) \cong Z^{\left(2^{n-1}+1\right)}
$$

If $g(k)=\{\mathbf{1},-\mathbf{I}\} \times h$, then the direct summands are generated by $\langle 1\rangle$, $\langle 1\rangle-\langle-1\rangle$, and all the elements $\langle 1\rangle-\langle a\rangle, a \in h$.

Proof. Propositions 1.22 and 1.19 show that the homomorphism $\prod j_{P}$ in (1.16) is in fact an injection. We shall show that the image of $\prod j_{P}$ contains $2^{n-1}$ linearly independent elements which will prove all the assertions of Proposition 1.23. Let $\left\{\boldsymbol{a}_{1}, \ldots, \boldsymbol{a}_{n-1}\right\}$ be any basis for $h$. The $2^{n-1}$ orderings of the field are completely determined by the signs of $a_{i}$. Tet $P$ be any ordering and assume that $\varepsilon_{1} a_{1}, \ldots, \varepsilon_{n-1} a_{n-1} \in P$, where $\varepsilon_{i}= \pm 1$. Put

$$
A_{P}=\left(\langle 1\rangle-\left\langle-\varepsilon_{1} a_{1}\right\rangle\right) \ldots\left(\langle 1\rangle-\left\langle-\varepsilon_{n-1} a_{n-1}\right\rangle\right)
$$

(we use here the ring structure of $G(k)$ ).

Observe that $j_{P}(\langle 1\rangle-\langle a\rangle)=0$ if $a \in P$ and $j_{P}(\langle 1\rangle-\langle a\rangle)=\langle 1\rangle-\langle-1\rangle$ if $-a \in P$.

Hence

and

$$
j_{P}\left(A_{P}\right)=(\langle 1\rangle-\langle-1\rangle)^{n-1}=2^{n-2}(\langle 1\rangle-\langle-1\rangle) \neq 0 .
$$

$$
j_{P^{\prime}}\left(A_{P}\right)=0 \quad \text { for any ordering } P^{\prime} \neq P .
$$

Thus the images under the map $\prod j_{P}$ of $2^{n-1}$ elements $A_{P}$, where $P$ runs through all the orderings of $k$, form a linearly independent subset of $\prod G_{0}\left(k_{P}\right)$, and by the remark above, we are finished.
2. Classification of Grothendieck groups for fields with $q \leqslant 8$. The main results in this section are Theorems 2.4 and 2.5 which classify the groups $G(k)$ for fields with $q=8$. For the sake of completeness we include the cases $q=1,2$, 4. Theorem 2.1. follows directly from Lemma 1.1 and Theorem 2.2 follows from Proposition 1.7 (the non-real casse) and Lemma 1.10 (the real ease). Theorem 2.3 has been proved first in [17] by another method and we give here a brief and ensy proof for it by using the results of $\S 1$.

TanonYm 2.1. Tot to be a field of charateristie other than 2 and $q(k)=1$. Then $G(7) \cong Z$ and the group is generated by the olass $\langle 1\rangle$.

Trusomidy 2.2. Let $t$ be a field of characteristic other than 2 and $q(7)=2$. If $k$ is non-real, then

$$
G(k) \cong \boldsymbol{Z} \oplus \boldsymbol{Z} / \mathbf{Z} \boldsymbol{Z} .
$$

If tis is real, then

$$
G(k) \cong \boldsymbol{Z} \oplus \boldsymbol{Z} .
$$

In both oases the oyolio summands are generated by $\langle 1\rangle$ and $\langle 1\rangle-\langle a\rangle$, where $a$ is a non-square in $k$.

Tronomin. 2.3. Let $k$ be a field of characteristio not 2 and $q(k)=4$. A. Let $k$ be a non-real field.

(I) If every binary quadratio form over th is universal, then

$$
G(k) \cong \boldsymbol{Z} \oplus(\boldsymbol{Z} / 2 \boldsymbol{Z})^{(2)} \text {. }
$$

(II) If (1, 1) is universal but there are non-universal binary forms over $k$, then

$$
G(h) \cong \boldsymbol{Z} \oplus(\boldsymbol{Z} / \mathbf{Z Z})^{(3)}
$$

(III) If $(1,1)$ is not universal, then

$$
G(k) \simeq \boldsymbol{Z} \oplus \boldsymbol{Z} / 4 \boldsymbol{Z} \oplus \boldsymbol{Z} / \mathbf{2} \boldsymbol{Z} .
$$

13. Let $k$ be a real fietd.

(IV) If to is wow-pythagorean, then

$$
Q(k) \cong Z^{(2)} \oplus \boldsymbol{Z} / 2 \boldsymbol{Z}
$$

(V) If $k$ is prythagorean, then

$$
G(l) \simeq Z^{(3)}
$$

Proof. Wo put $g(k)=\{\mathbf{1}, \boldsymbol{e}\} \times\{\mathbf{1}, \boldsymbol{a}\}$, where $e=-1$ unless $-\mathbf{1}$ is a square in $\%$. We also use the notation: $D=\langle 1\rangle-\langle e\rangle, A=\langle\mathbf{1}\rangle-\langle a\rangle$, $D=\langle 1\rangle-\langle e a\rangle$. If $\dot{q}=4$, then $s=1,2$ or infinity, as proved by Kaplansky ([5], Theorem 4). 
Case (I). Proposition 1.7 yields all the information needed:

$$
G_{0}=[E] \oplus[A] \cong(\boldsymbol{Z} / 2 \boldsymbol{Z})^{(2)}
$$

Case (II). By Lemma 1.I, we have $G_{0}=[E, A, D]$. Now $E$ and $A$ are linearly independent (Lemma 1.6) and $D$ does not belong to $[E, A]$, for otherwise $D=E+A$, i.e. $\langle 1, e a\rangle=\langle e, a\rangle$ and $\langle 1, e a\rangle$ would be a second universal binary class, contrary to Corollary 1.9. Hence

$$
G_{0}=[E] \oplus[A] \oplus[D] \cong(\boldsymbol{Z} / 2 \boldsymbol{Z})^{(3)} .
$$

Case (III). By Corollary 1.3, the group $G_{0}$ is generated by $E$ and $A$. Here $2 E=0$ and $2 A \neq 0$ (otherwise $\langle 1,1\rangle=\langle a, a\rangle$ would be a universal olass). Moreover, $\langle 1,1\rangle=\langle-1,-1\rangle$ and $\langle a, a\rangle=\langle-a,-a\rangle$, bence $\langle 1,1,1,1\rangle=\langle-1,-1,1,1\rangle=\langle a,-a, a,-a\rangle=\langle a, a, a, a\rangle$, and. so $4 A=0$. Now $E$ does not belong to [:A] since none of the elements of [A] has determinant - 1 . Thus

$$
G_{0}=[A, E]=[A] \oplus[E] \cong \boldsymbol{Z} / 4 \boldsymbol{Z} \oplus \boldsymbol{Z} / 2 \boldsymbol{Z} .
$$

Oase (IV). Now $(1,1)$ represents a non-square in $k$, so assume that $(1,1) \approx a$. Then $(1,-a)$ is universal and Proposition 1.11 applies:

$$
G(k)=[\langle 1\rangle] \oplus[E] \oplus[A] \cong \boldsymbol{Z} \oplus \boldsymbol{Z} \oplus \mathbf{Z} / 2 \boldsymbol{Z}
$$

Oase $(\nabla)$. Now Proposition 1.17 shows that

$$
G(k)=[\langle 1\rangle] \oplus[\mathbb{E}] \oplus[A] \cong \mathbf{Z}^{(3)} .
$$

We come now to the main theorems of this section. We recall our notation: $q=q(k)=|g(k)|, q_{2}=|D(1,1)|, u_{2}=\left|U_{2}(k)\right|$, the number of binary universal classes over $k$. Both $q_{2}$ and $u_{2}$ are powers of two, $q_{2} \leqslant q, u_{2} \leqslant q$ and $q_{2}>1$ if $k$ is non-real and $q>1$. It will be also convenient to have the inequality $s \leqslant q_{2}$ at hand ([11], p. 126).

THEOREM 2.4 (Classifieation Theorem for Grothendieck groups of non-real fields with $q=8$ ). Let $k$ be a non-real field of oharacteristic not 2 and $q(k)=8$. Then the Grothendieek group $G(k)$ is determined as follows.

(i) The case $q_{2}=8$.

(I) If $u_{2}=8$ (i.e. every binary quadratic form over $k$ is universal), then $G(k) \cong \mathbf{Z} \oplus(\mathbf{Z} / 2 \mathbf{Z})^{(3)}$

(II) If $2 \leqslant u_{2}<8$, then

$$
G(k) \cong \boldsymbol{Z} \oplus(\boldsymbol{Z} / 2 \boldsymbol{Z})^{(4)}
$$

(III) If $u_{2}=1$ and there exists a non-universal binary form representing more than 2 elements of $g(7)$, then

$$
G(k) \cong \boldsymbol{Z} \oplus(\boldsymbol{Z} / 2 \boldsymbol{Z})^{(5)} .
$$

(IV) If $u_{2}=1$ and every non-universal binary form represents at most 2 elements of $g(k)$, then

$$
G(k) \cong \boldsymbol{Z} \oplus(\boldsymbol{Z} / 2 \boldsymbol{Z})^{(7)}
$$

(ii) The oase $q_{2}=4$.

(V) If $s=4$, or if $s \leqslant 2$ and $u_{2} \geqslant 2$, then

$$
Q\left(k_{i}\right) \cong \boldsymbol{Z} \oplus \boldsymbol{Z} / \boldsymbol{\Delta} \boldsymbol{Z} \oplus(\boldsymbol{Z} / 2 \boldsymbol{Z})^{(2)} .
$$

(VI) If $s: 2$ and $u_{2}=1$, then

$$
G(k) \cong \boldsymbol{Z} \oplus \boldsymbol{Z} / \mathbf{A} \oplus(\boldsymbol{Z} / 2 \boldsymbol{Z})^{(3)}
$$

(iii) The oase $q_{2}=2$.

(VII) If $q_{2}=2$, then every anisotropic binary form represents exactly 2 elements of $g\left(l_{c}\right)$ and

$$
G(k) \cong \boldsymbol{Z} \oplus(\boldsymbol{Z} / \mathbf{4} \boldsymbol{Z})^{(3)} \oplus \boldsymbol{Z} / 2 \boldsymbol{Z} .
$$

Remark. In Section 4 we produce examples of fields which show that all the cases of Theorem 2.4 do occur, except possibly (II).

Trnoxwm 2.5 (Olassification Theorem for Grothendieck groups of real fields with $q=8$ ). Let $k$ be a real field with square etass number $q=8$. Then the Grothendiecti group $Q(k)$ is determined as follows.
(i) The case $q_{2}=4$

(I) If $u_{2} \geqslant 2$, then

(II) If $u_{2}=1$, then

$$
G(k) \cong Z^{(2)} \oplus(\boldsymbol{Z} / 2 \boldsymbol{Z})^{(2)}
$$

$$
G(7) \cong Z^{(2)} \oplus(\mathbf{Z} / 2 \mathbf{Z})^{(3)}
$$

(ii) The case $q_{2}=2$

(III) If $u_{2} \geqslant 2$, then

$$
G(k) \cong Z^{(3)} \oplus \boldsymbol{Z} / 2 \boldsymbol{Z}
$$

(IV) $x f u_{2}=1$, then

$$
G(k) \cong \boldsymbol{Z}^{(3)} \oplus(\boldsymbol{Z} / 2 \boldsymbol{Z})^{(2)}
$$

(iii) The wase $g_{0}=1$ (i.e., 7 is a pythagorean fietd).

(V) If there is an anisotropio binary form representing more than 2 elements of $g(k)$, then

$$
G(k) \cong \boldsymbol{Z}^{(4)}
$$

(VI) If every anisotropic binary form represents at most 2 elements of $g(k)$, then

$$
\theta(k) \cong \mathbf{Z}^{(5)}
$$


Remark. We shall prove in Section 4 that each of the six eases of Theorem 2.5 actually occurs, except possibly (II).

Proof of Theorem 2.4. In each of the seven cases we fix a decomposition of $g(k)$ into direct product of subgroups of order two: $g\left(l_{i}\right)=\{\mathbf{I}, \boldsymbol{e}\} \times\{\mathbf{I}, \boldsymbol{a}\} \times\{\mathbf{I}, \boldsymbol{b}\}$, where we assume that $e=-1$ whenever -1 is not a square in $k$, and $a$ and $b$ are chosen to satisfy certain comditions. We use the following notation for the generators of $\theta_{0}(k\rangle$ : $E=\langle 1\rangle-\langle e\rangle, A=\langle 1\rangle-\langle a\rangle, B=\langle 1\rangle-\langle b\rangle, C=\langle 1\rangle-\langle a b\rangle, D=\langle 1\rangle-$ $-\langle e a\rangle, F=\langle 1\rangle-\langle e b\rangle, H=\langle 1\rangle-\langle e a b\rangle$.

Case (i) $q_{2}=8$. Here the form $(1,1)$ is universal and so the stufe $s \leqslant 2$. Also $\langle 1,1\rangle=\langle c, c\rangle$ for every $\boldsymbol{c}$ in $g(k)$, so that $2(\langle 1\rangle-\langle\theta\rangle)=0$.

Subcase (I). Proposition 1.7 proves that

$$
G_{0}=[E] \oplus[A] \oplus[B] \cong(\boldsymbol{Z} / 2 \boldsymbol{Z})^{(3)} .
$$

Subcase (II). Choose a non-square $e$ such that $(1, e)$ be universal. Then by Lemma 1.2, we have $Q_{0}=[E, A, B, O]$. Assume that $C \in[D$, $A, B]$; then necessarily $O=A+B$ (determinants), and this implies $\langle 1, a b\rangle=\langle a, b\rangle$. We show that also $(1, a b) \approx e$. In fact, if $s=1$, then from $(1, e) \approx a b$ we get $(1, a b) \approx e$. If $s=2$, then $e=-1,(1,1) \approx-a b$ and so $(1, a b) \approx-1=e$. Thus in either case $(1, a b) \approx e, a, b$ and so $\langle 1, a b\rangle$ is a third universal class, contrary to Corollary 1.9. (An alternative proof: if $O=A+B$, then $G_{0} \cong(\boldsymbol{Z} / 2 \boldsymbol{Z})^{(3)} \cong g(k)$; and by Theorem 2.2 of [1.8] all binary forms over $k$ have to be universal). Hence using Lemma 1.6 we get

$$
G_{0}=[B, A, B, O]=[E] \oplus[A] \oplus[B] \oplus[O] \cong(\boldsymbol{Z} / 2 \boldsymbol{Z})^{(4)} .
$$

Subcase (III). $(1,1)$ is the only universal form, so $\langle 1,1\rangle=\langle 1,-1\rangle$ and consequently $s=1$. Now $D(1, c)$ is a subgroup of $g(k)$ and $|D(e, d)|$ $=|D(1, c d)|$. Fence if $2<|D(c, d)|<8$, then $|D(c, d)|=4$, and so there exists a form $(1, e)$ representing exactly 4 elements of $g(k)$. If $D(1, e)$ $=\{\boldsymbol{1}, \boldsymbol{e}, \boldsymbol{a}, \boldsymbol{e} \boldsymbol{a}\}$, then we choose an element $\boldsymbol{b}$ in $g(k)$ which is not represented by $(\mathbf{1}, e)$ and write $g(k)=\{\mathbf{1}, \boldsymbol{e}\} \times\{\mathbf{1}, \boldsymbol{a}\} \times\{\mathbf{1}, \boldsymbol{b}\}$. We know that $\langle 1, e\rangle=\langle a, e a\rangle$, and this implies $\langle b, e b\rangle=\langle a b, e a b\rangle$ and $\langle 1, a\rangle=\langle e, e a\rangle$. Thus $E=A+D$ and $B+F=O+H$. The group $G_{0}$ is generated by the elements $E, A, B, O, D, F, H$ (Lemma 1.1) and the above relations show that $D$ and $H$ are superfluous in the set of generators. By Lernma, 1.6, the elements $E, A, B$ are independent; put $G_{1}=[B, A, B]$. Then $O$ does not belong to $G_{1}$, for otherwise $O=A+B$ and $\langle 1, a\rangle=\langle b, a b\rangle$. We observe that $(1, e) \approx a$ implies $(1, a) \approx e$ (since $s=1$ ), and so $(1, a)$. would be a universal form which is "not the case. Thus $O \& G_{1}$.

Write $G_{2}=[E, A, B, O]$ and observe that $F_{\phi} G_{2}$. Otherwise, comparing determinants we get either $F=E+B$ or $\dot{F}=E+A+C$. Now the first possibility gives $\langle 1, e\rangle=\langle b, e b\rangle$ which implies the universality of $(1, e)$, a contradiction. The second possibility implies $\langle 1, b\rangle=\langle e a, e a b\rangle$. But this is also impossible: if $(1, b) \approx e a$, then $(1, e a) \approx b$ and from $(1, e) \approx e a$, $(1, a) \approx e a$ wo geti $(1, e a) \approx a, e$. Thus $(1, e a)$ would be a second universal binary form. Summarizing we get:

$$
\begin{aligned}
G_{0} & =G_{2} \oplus\left[W^{\top}\right]=G_{1} \oplus[G] \oplus\left[F^{\prime}\right] \\
& =[W] \oplus[A] \oplus[B] \oplus[O] \oplus\left[W^{\prime}\right] \cong(Z / 2 Z)^{(5)} .
\end{aligned}
$$

Subcase (IV). Fero againi $s=1$ and $G_{0}$ is generated by the seven elements $I I, A, B, C, D, F, H$. Wo prove that they are independent. Let us prove tirst that any four of them are independent. Suppose, for instance, $A+B+D+F=0$. Then $A+B=D+F$, hence $\langle a, b\rangle=\langle e a$, $e b\rangle$ and $(a, b)$ represents at least 4 olements of $g(k\rangle$, contrary to (IV). Similarly, any sum of three or two of them is not 0 . Put $G_{1}=[E, A, B, C]$ and $G_{2}=[D, I, H]$. To get the result it suffices to show that $G_{1} \cap G_{2}=0$. First note that none of $D, F, H$ belongs to $G_{1}$, for $\operatorname{det}(E+A+B+C)=\boldsymbol{e}$ and so this sum is not equal to $D, F, H$, and as remarked above, $D, F, H$ cannot be expressed as a sum of 3 or less generators of $G_{1}$. Similarly, $D+F, D+H, H^{3}+H$ do not belong to $G_{1}$. To see this observe that the determinants of the sums of any three of $E, A, B, C$ are $\boldsymbol{e a b}, \boldsymbol{e} \dot{a}, \boldsymbol{e b}, \mathbf{1}$. Thus none of $D+F, D+H, T+H$ can be an sum of three or four generators of $G_{2}$ because of unequal determinants, and also none of them can be a sum of less than 3 generatiors of $G_{1}$. It remains to prove that $D+F+H \& G_{1}$. First observe that det $(D)+F+F)=\boldsymbol{e}$ and none of the sums of two or three generations of $G_{1}$ has determinant $e$. Thus the only possibility is $B+A+B+C=D+Z+F$, or equivalentily, $H=B+A+B+C+D+F$ or else $\langle 1,1,1,1,1, e a b\rangle=\langle e, a, b, a b, e a, e b\rangle$. That this cannot happen is ensily seen on using Witt's theorem on piecewise equivalence ([19], Satz 7; compare [7], p. 25). In view of the hypothesis (IV) every dyadic change of the form $(e, a, b, a b, e a, e b)$ reduces merely to a permutation of the diagonal entries and so we never pass from this form by dyadic changes to (1., 1, 1, 1., 1, eab). Thus we have proved that $\theta_{1} \cap G_{2}=0$, and so wo get

$$
Q_{0}=\ldots Q_{1}\left(A Q_{2}=[D] \oplus[A](B)[B] \oplus[O] \oplus[T)\right] \oplus\left[H^{\top}\right] \oplus[H] \cong(\boldsymbol{Z} / 2 \boldsymbol{Z})^{(7)} \text {. }
$$

(anse (ii). $q_{2} \leq 4$. We know that $s, q_{2}$, so we have now $s \leqslant 4$ and $s>1$ (otherwise $q, \cdots q, \cdots, 8$ ). Note thlso that in the catse (ii) the class 4 ( 1 ) is universal. This is obvious if $s=2$, and if. $s=4$, then $(1,1)$ is anisotropio and representis 4 elements of $g(k)$, hence $(1,1,1)$ represents at least 5 (3) ementis of $g(k)$ (e.. [9], p. 13). Consequently, the multiplicative class $4\langle 1\rangle$ represents all $g(k)$. Hence $4(\langle x\rangle-\langle 0\rangle)=0$, for every $c$ in $k^{*}$.

Subcuse (V). Consider first the case $s=4$. Assume $-1=a+b$ whore $a, b \in D(1,1)$. These lie in difforent cosets of $k^{*}$ modulo $k^{* 2}$ and so wo may writie $D(1,1)=\{\mathbf{1}, \boldsymbol{a}\} \times\{\mathbf{1}, \boldsymbol{b}\}$ and $g(k)=\{\mathbf{1},-\mathbf{1}\} \times D(\mathbf{1}, 1)$. 
Here $\langle a, b\rangle=\langle-1,-a b\rangle$, hence $\langle 1, a, b, a b\rangle=\langle 1,-1,1,-1\rangle$ which is equivalent to $A+B+O=2 E$. By Lemma 1.2, $G_{0}=[E, A, B, C]$ and the above relation shows that

$$
G_{0}=[E, A, B]=[B] \oplus[A] \oplus[B] \cong \boldsymbol{Z} / 4 \boldsymbol{Z} \oplus(\boldsymbol{Z} / 2 \boldsymbol{Z})^{(2)} .
$$

Assume that $s=2$. Now $D(1,1)=\{\mathbf{1},-\mathbf{I}\} \times\{\mathbf{I}, \boldsymbol{a}\}$ and $g\left(T_{c}\right)=D(1,1) \times$ $\times\{\mathbf{I}, \boldsymbol{b}\}$. Consider (V). Since $u_{2} \geqslant 2$, either $(1, a)$ or $(1,-a)$ is universal and we choose $a$ so that $(1, a)$ be universal. By Lomma 1.4. we obtain $G_{0}=[E, A, B]$. Here $2 E=2 A=4 B=0,2 B \neq 0$ and $E \phi[A, B], A \notin[B]$ (by comparison of determinants). Hence

$$
G_{0}=[B] \oplus[E] \oplus[A] \cong \boldsymbol{Z} / 4 \boldsymbol{Z} \oplus(\boldsymbol{Z} / 2 \boldsymbol{Z})^{(\underline{q})} .
$$

Subcase (VI). Now $\langle 1,-1\rangle$ is the only universal class and $G_{0}=[E, A$, $B, O]$ (Corollary 1.3). From $\langle 1,1\rangle=\langle a, a\rangle$ we obtain $2 B=2 O$. Hence $G_{0}=[E, A, B, B-C]$, where $2 E=2 A=2(B-C)=0$ and $4 B=0,2 B \neq 0$. Certainly $B \& G_{1}=[A, B, B-C]$ (determinants), so we may write $G_{0}$ $=[E] \oplus G_{1}$. Now $[B] \cap[A, B-C]=0$, otherwise we would get $2 B$ $=A+B-C$ (determinants), and $\langle 1, a\rangle=\langle b, a b\rangle$, contrary to the nonwniversality of $(1, a)$. Thus

$$
\theta_{1}=[B] \oplus[A, B-C]=[B] \oplus[A] \oplus[B-O],
$$

since $A \neq B-C$ (otherwise $(1,-a)$ would be universal). Hence

$$
G_{0}=[B] \oplus[E] \oplus[A] \oplus[B-O] \cong \boldsymbol{Z} / 4 \boldsymbol{Z} \oplus(\boldsymbol{Z} / 2 \boldsymbol{Z})^{(3)} .
$$

Oase (iii). $q_{2}=2$. Now obviously $s>1$ and from $s \leqslant q_{2}$ we get $s=2$. Thus $D(1,1)=\{\mathbf{1},-\mathbf{1}\}$. None of anisotropic binary forms can be universal, since if $(1, a)$ is universal, then $-a_{\in} D(1,1)$. But, in fact, more is true. Using an argument due to L. Szczepanils, we prove that $|D(e, d)| \leqslant 2$ for any anisotropic form $(e, d)$. If this is not the case, we may assume that $|D(1, a)|=4$ and $D(1, a)=\{\mathbf{I}, \boldsymbol{a}, \boldsymbol{b}, \boldsymbol{a b}\}$. Then from $\langle 1,1\rangle$ $=\langle-1,-1\rangle$ we get $\langle 1, b, b\rangle=\langle 1,-b,-b\rangle$. We have $\langle 1,-b\rangle \approx-a$ and so $\langle 1,-b,-b\rangle \approx-a$, while we shall prove that $\langle 1, b, b\rangle$ does not represent $-a$. To this end we must determine $D(1, b, b)=\bigcup D(a, b)$, where $\boldsymbol{c}$ runs through $D(1, b)$. We shall show that $D(1, b)=\{:=\{, \boldsymbol{1}\}$ and so $D(1, b, b)=D(1, b) \cup D(b, b)=\{\mathbf{I}, \boldsymbol{b},-\boldsymbol{b}\}$ does not contain --a, as required.

Now we determine $D(1, b)$. Knowing $D(1,1)$ and $D(1, a)$ we see at once that $-\mathbf{I}$ and $-\boldsymbol{a}$ do not belong to $D(1, b)$, hence also $-\boldsymbol{b}$ and $-\boldsymbol{a b}$ do not. If $\boldsymbol{a b} \in D(\mathbf{1}, b)$, then $(1,-a b) \approx-a,-b,-a b$, hence also $(1,-a b)$ $\approx(-a)(-a b)=a^{2} b$. Thus $(1,-a b) \approx a^{2} b(-b)$, and so $a b \in D(1,1)$, a contradiction. Hence $\boldsymbol{a} b \notin D(1, b),|D(1, b)|<4$, and so $D(1, b)=\{\mathbf{1}, \boldsymbol{b}\}$ as required.
Now we determine $G(k)$. Write $g(k)=\{\mathbf{1},-\mathbf{1}\} \times\{\mathbf{1}, \boldsymbol{a}\} \times\{\mathbf{1}, \boldsymbol{b}\}$; then $2 E=4 A=4 B=4 C=0$ and $2 A \neq 0,2 B \neq 0,2 C \neq 0$. By Lemma 1.2,

$$
Q_{0}=[E, A, B, O]=[E] \oplus[A, B, C]
$$

(since $E \phi[A, B, O]$ ). Here $[A] \cap[B]=0$, since otherwise $2 A=2 B$ which gives $\boldsymbol{a b} \in D(1,1)$, and we want to prove that $[C] \cap[A, B]=0$. Indeed, if not, then oliminating the casss, where determinants are not equal, ono gots tho following possibilities: either

$$
\text { (1) } 2 O=2 A+2 B
$$

or

(2) $C$ or $3 C=A+B$ or $3 A+B$ or $A+3 B$ or $3 A+3 B$.

Consider (1). Wo obtain $\langle 1,1, a b, a b\rangle=\langle a, a, b, b\rangle$ and we show that this is impossible by applying piecewise equivalence. Observe that $(a, a)$ can be changed dyadically only into $(-a,-a)$ and similarly $(b, b)$ only into $(-b,-b)$. Now $(a, b),(-a, b)$ etc. represent only 2 elements of $g(k)$ and so cannot be changed dyadically in a non-trivial manner. Hence there is no way of getting either 1 or $a b$ by applying dyadic changes to $(a, a, b, b)$ and consequently (1) cannot hold.

As to (2), we observe that the possibilities are $\pm O= \pm A \pm B$. Here the only one non-obvious possibility is $A+B+C=0$ (all the others are ruled out by $|D(e, d)| \leqslant 2, \boldsymbol{e d} \neq-\mathbf{1})$. But this implies $\langle 1,1,1\rangle$ $=\langle a, b, a b\rangle$ which again contradicts piecewise equivalence. Hence

$$
Q_{0}=[A] \oplus[B] \oplus[\sigma] \oplus[\Phi] \cong(\boldsymbol{Z} / 4 \boldsymbol{Z})^{(3)} \oplus \boldsymbol{Z} / 2 \boldsymbol{Z} .
$$

This completes the proof of Theorem 2.4.

Proof of Theorem. 2.5. Let $k$ be a real field with $q=8$. In each of the six cases of the theorem we fix an ordering of $k$ and write $g(k)$ $=\{\mathbf{1},-\mathbf{1}\} \times\{\mathbf{1}, \boldsymbol{a}\} \times\{\mathbf{1}, \boldsymbol{b}\}$, where $a$ and $b$ are assumed to be positive in the given ordering of $k$. We donote the generators of $G_{0}$ by $E=\langle\mathbf{1}\rangle-$ $-\langle-1\rangle, A=\langle 1\rangle \cdots\langle a\rangle$, etce, as in the proof of the preceding theorem. Note that now $W$ is of infinite order and by Lemman 1.10, we have

$$
Q_{0} \ldots[E] \oplus Q_{1}
$$

where $G_{1}=[A, B, C]$ is to be determined.

Oase (i). $q_{2}=4$.

Assume $(\mathbf{I})$. Tu this case $\boldsymbol{a}, \boldsymbol{b} \in D(1,1)$ and $\langle 1,-a\rangle,\langle 1,-b\rangle$ are easily shown to be universat. Tfence by Proposition 1.11,

$$
Q_{1}::[A, B]:=[A] \oplus[B] \cong(\boldsymbol{Z} / 2 \boldsymbol{Z})^{(2)} \text {. }
$$


If there is only one universal binary class, then $A+B+C \neq 0$ and we obtain

$$
G_{1}=[A, B, C]=[A] \oplus[B] \oplus[\dot{O}] \cong(\boldsymbol{Z} / 2 \boldsymbol{Z})^{(3)},
$$

which proves $(\Pi)$.

Case (ii). $q_{2}=2$.

Assume $a$ is a sum of two squares and $b$ is not. If an element $c$ is a sum of squares in $k$, then it is already a sum of two squares. Indeed, let $t$ be the minimal number such that any sum of squares in $t$ is a sum of $t$ squares and $2^{\tau} \leqslant t<2^{\tau+1}$. Then $q \geqslant 2 \cdot 2^{x(\tau+1) / 2} \geqslant 2 t$ ([11], Satz 25), "and it follows easily that in case $q=8$ we have $t=2$. A consequence of this fact is that $2 A=0$ and $B$ and $O$ are of infinite order.

Assume now (III). We may assume that $(1,-a)$ is universal. Hence $(1,-a) \approx-b$ and $\langle 1, b\rangle=\langle a, a b\rangle$, i.e. $B=A+C$. Hence

$$
G_{1}=[A, B]=[B] \oplus[A] \cong \boldsymbol{Z} \oplus \boldsymbol{Z} / 2 \boldsymbol{Z} .
$$

Subcase (IV). We have $\langle 1,1\rangle=\langle a, a\rangle$, hence $\langle b, b\rangle=\langle a b, a b\rangle$ and $2 B=2 C$. Now $\theta_{1}=[B, A, B-C]$, where $B$ is of infinite order and $2 A=2\langle B-C)=0$. Hence $G_{1}=[B] \oplus[A, B-C]$. Now $A \neq B-C$, since otherwise $\langle 1, b\rangle=\langle a, a b\rangle,(1,-a) \approx-b$ and so $(1,-a)$ would be a seeond universal form, contrary to (IV). Thus

$$
G_{\mathbf{1}}=[B] \oplus[A] \oplus[B-C] \cong \boldsymbol{Z}_{\oplus}(\boldsymbol{Z} / 2 \boldsymbol{Z})^{(2)} .
$$

Case (iii). $q_{2}=1$.

Proposition 1.19 asserts that $G(k)$ is a torsion free group and so $A$, $B, O$ are all of infinite order.

Assume $(\nabla)$. Without loss of generality let $(1, a)$ represent more than 2 elements of $g(k)$, i.e. $(1, a) \approx b$. Hence $\langle 1, a\rangle=\langle b, a b\rangle$ and $A=B+C$ and $G_{1}=[A, B]$. Now Proposition 1.21 proves that

$$
G_{1}=[A] \oplus[B] \cong Z^{(2)} \text {. }
$$

Subcase (VI). By Proposition 1.22, the field is smperpythagorean and now Proposition 1.23 proves that

$$
G_{1}=[A] \oplus[B] \oplus[C] \cong Z^{(3)}
$$

This completes the proof of Theorem 2.5.

3. Classification of Witt groups for fields with $q \leqslant 8$. The Witt grotp $W(k)$ of a field $k$ can be defined as the factor group $G(k) / H$, where $H$ is the subgroup of $G\left(k_{i}\right)$ generated by the hyperbolic plane $\langle 1,-1\rangle$. The canonical surjection $f: G\left(k_{k}\right) \rightarrow W(k)$ can be easily shown to act in the following manner:

$$
f\left(\left\langle a_{1}, \ldots, a_{n}\right\rangle-\left\langle b_{1}, \ldots, b_{m i}\right\rangle\right)=\left\langle a_{1}, \ldots, a_{n},-b_{1}, \ldots,-b_{m}\right\rangle \bmod \mathbb{Z} .
$$

Fence all the results of Section 1 coneerning sets of generators for $G(k)$ can be reformulated for the case of Witt groups. If $G(\bar{k})=X_{1} \oplus \ldots \oplus X_{n}$, then $W(k)=f\left(X_{1}\right)+\ldots+f\left(X_{n}\right)$ and if $H \subset X_{1}$, then $W\left(k_{i}\right)=f\left(X_{1}\right) \oplus \ldots$ $\ldots \oplus f\left(X_{n}\right)$, where $f\left(X_{1}\right) \cong X_{1} / H$ and $f\left(X_{i}\right) \cong X_{i}$ for $i>1$.

Hence, to obtain a classification for Witt groups of fields with $q \leqslant 8$ we shall use the classification of Grothendieck groups earried out in Section 2. In each of the cases we find a direct stmmand $X$ of $G(k)$ such that $H \subset X$, snd we determine a direct sum decomposition for $X / H$ (Proposition 3.1.) and then write antomatically a direct sum decomposition for $W(\%)$. Observe that for $X$ we can take $[\langle 1\rangle] \oplus[\langle 1\rangle-\langle-1\rangle]$, whenever $\langle 1\rangle-\langle-1\rangle$ gonerates an dixect summand of $G(7)$, since $\langle 1,-1\rangle=2\langle 1\rangle-$ $-(\langle 1\rangle-\langle-1\rangle)$. It titrms out that $\langle 1\rangle-\langle-1\rangle$ generates a direct summand for all fields $k$ with $g \leqslant 8$, as can be seen inmediately from the decompositions of $G(k)$ given in Section 2. By Lemna 1.10, this happens for all real fjelds and for non-real fields one can easily prove that $\langle 1\rangle-\langle-1\rangle$ generates a direct summand of $G_{0}(k)$ in each of the following cases (independently on the value of $q$ ): (i) $s=1$ or 2 , (ii) $s>2$ and the class $s\langle 1\rangle$ is universal (then $\langle 1\rangle-\langle-1\rangle$ is an element of maximal order in $G_{0}\left(F_{i}\right)$ and generates a direct summand by [16], Oorollary 2)(1).

Proposinos 3.1. Suppose $\langle 1\rangle-\langle-1\rangle$ generates a direct summand of $G_{0}\left(k_{0}\right), X=[\langle 1\rangle] \oplus[\langle\mathbf{L}\rangle-\langle-1\rangle]$ and $H=[\langle 1,-1\rangle]$

If $t_{i}$ is a non-real field and $s$ is the stufe of $k$, then $X / H \cong Z / 2 s Z$ and the group is generated by $\langle 1\rangle \bmod H$.

If $t$ is a reat fietd, then $X=[\langle 1\rangle] \oplus H$, and so $X / H \cong Z$, where the group is generated by $\langle 1\rangle \bmod H$.

Proof. In the real case there is nothing to prove and so we assume that $k$ is non-real. Wo define $h: X \rightarrow \boldsymbol{X} / 2 s \boldsymbol{Z}$ by putting

$$
h(x\langle 1\rangle+m(\langle 1\rangle-\langle-1\rangle))=(x+2 m)(\bmod 2 s) .
$$

Clearly $h$ is a surjective homomorphism and Ker $h$ can be easily shown to. coincido with $H$. Tenco the result.

Tronoxism 3.2 (Classification Theorem for Witt groups of fields with $q \leqslant 8)$. tet th be a field of oharasteristio other than 2 and with square class number 458 . Then the Witt growp $W($ k $)$ is determined as follows.

1. $q=1: W(k): \because \boldsymbol{Z} / 2 \boldsymbol{Z}$.

2, $q=-2$, n nom real:

(2.1) $\quad W^{\prime}(k) \backsim(\boldsymbol{Z} / 2 \boldsymbol{Z})^{(2)}, \quad$ if $s=1$,

(2.2) $\therefore \boldsymbol{Z} / 4 \boldsymbol{Z}, \quad$ if $8=2$.

(1) Added in proot. If ean bo proved that $\langle\mathrm{I}\rangle-\langle-1\rangle$ always generates a direet sumunand in $G_{0}(t)$. The prooll will appear in the Proce of $J$. Bolyai Math. Soc. Colloquitum on Nrumber 'lheory held at Debrecen in October 1974. 


$$
q=2,7 \text { real: }
$$

(2.3) $\quad W(k) \cong Z$.

3. $q=4$, z non-real:

(3.1) $\quad W(k) \cong(\boldsymbol{Z} / 2 \boldsymbol{Z})^{(3)}$

if (I) of Theorem 2.3 holds and $s=1$,

4. $q=8, k$ non-real:

$$
\begin{aligned}
& \cong Z / 4 Z \oplus \boldsymbol{Z} / \mathbf{Z Z} \\
& \cong(\boldsymbol{Z} / 2 \boldsymbol{Z})^{(\mathbb{d})} \text {, } \\
& \cong(\boldsymbol{Z} / 4 \boldsymbol{Z})^{(2)} \text {, } \\
& \text { if (II) holds, } \\
& \text { if (MI) holds. }
\end{aligned}
$$$$
\text { (I) holds and } s=2 \text {, }
$$

$q=4$, k real:

$W(k) \cong \boldsymbol{Z} \oplus \boldsymbol{Z} / 2 \boldsymbol{Z}$,

if $k$ is non-pythagorean,$$
W(k) \cong(\boldsymbol{Z} / 2 \boldsymbol{Z})^{(4)},
$$$$
\cong \boldsymbol{Z} / 4 \boldsymbol{Z} \oplus(\boldsymbol{Z} / 2 \boldsymbol{Z})^{(2)} \text {, }
$$$$
\simeq(\boldsymbol{Z} / 2 \boldsymbol{Z})^{(5)} \text {, }
$$$$
\simeq \boldsymbol{Z} / 4 \boldsymbol{Z} \oplus(\boldsymbol{Z} / 2 \boldsymbol{Z})^{(3)} \text {, }
$$$$
\cong(\boldsymbol{Z} / 2 \boldsymbol{Z})^{(6)} \text {, }
$$$$
\simeq(\boldsymbol{Z} / 2 \boldsymbol{Z})^{(8)} \text {, }
$$

5. $q=8, k$ real:

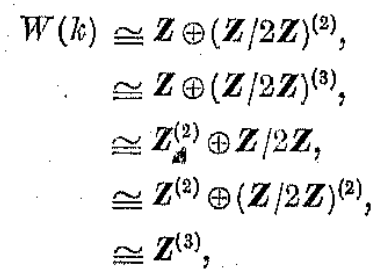

$$
\cong \mathbf{Z}^{(4)}
$$

if $q_{2}=u_{2}=8$ and $s=1$,

if $q_{2}=u_{2}=8$ and $s=2$,

if $q_{2}=8,2 \leqslant u_{2}<8$ and $s=1$,

if $q_{2}=8,2 \leqslant u_{2}<8$ and $s=2$,

if $q_{2}=8, u_{2}=1$ and there exists $a \in l^{*}$ such that $\left.\mid D(1, a)\right\}=4$,

if $q_{2}=8, u_{2}=1$ and $|D(1, a)| \leqslant 2$ for every non-universal form $(1, a)$,

$\cong(\boldsymbol{Z} / 4 \boldsymbol{Z})^{(2)} \oplus \boldsymbol{Z} / 2 \boldsymbol{Z}, \quad$ if $q_{2}=4, u_{2} \geqslant 2$ and $s=2$,

$\cong \boldsymbol{Z} / 8 \boldsymbol{Z} \oplus(\boldsymbol{Z} / 2 \boldsymbol{Z})^{(2)}, \quad$ if $q_{2}=4$ and $s=4$,

$\cong(\boldsymbol{Z} / 4 \boldsymbol{Z})^{(2)} \oplus(\boldsymbol{Z} / 2 \boldsymbol{Z})^{(2)}$, if $q_{2}=4, u_{2}=1$ and $s=2$,

$$
\cong(\boldsymbol{Z} / 4 \boldsymbol{Z})^{(4)}, \quad \text { if } q_{2}=2 \text {. }
$$

Remark. The fields in all the cases are known to exist except in $(4.3),(4.4),(4.7)$ and $(5.2)$.

4. Examples. In this section wo give examples of fields corresponding to the Classification Theorems 2.4 and 2.5. For each case, except 2.4. II and 2.5. II, wo point ont a field which satisfies the conditions imposed in Classification Theorems. Let us remark that in [17] there have been given examples of fields showing that all the cases of Theorems $2.1,2.2$ and 2.3 aetially occur.

Wo shall use four different constructions of fields with finite square class mumber: Gross and Fischor's, fortanl power series fields over a field with finite square cless numtser, quadratic extensions of fields with finite, square alass number and a construction of Elman and lam. First we tate tho result of: Gross and Fischor ([4], pp. 301-302).

Lilmma 4.x. Let $k$ be any field of oharacteristic other than two and let $\left\{a_{i} \in k^{*}: i \in I\right\}$ be any set of representatives for a subgroup $S$ of the group $g(k)=\pi * / 7 * * 2$. Then there exists an algebraic extension $K$ of $k$ such that $\left\{a_{i}: i \in I\right\}$ is a set of representatives for elements of the group $g(K) . K$ can always be chosen non-real and if $k$ is real and $-1 \in S$, then $K$ can be ohosen real, too.

This lomma enables us to construct fields of the type 2.4.I and 2.5.T. In fact, twing Temma 4.1., one can prove that for any integer $n \geqslant 0$ there existis a field $k$ such that $q=w_{2}=2^{n}$ (i.e. every binary form over 7 is universal; see [1.], p. 407; [3], (3.8); [18], Theorem 2.3). This comprises 2.4..I,

The case 2.5.X. Wo shall puove that for any integer $n>0$ there exists a real field $k$ such that $q=2^{n}, q_{2}=\dot{u}_{2}=2^{n-1}$.

For $n=3$ we shall obtain a field satisfying 2.5.I. In the case $n=1$ We can put $k=\boldsymbol{R}$, so assume $n>1$. We choose $n$ prime numbers $p_{1}, \ldots, p_{n}$ such that $p_{i}=1$. (mod 4$), i=1, \ldots, n$ and the g.c.d. $\left(p_{i}+p_{j}, p_{1} \ldots p_{n}\right)=1$ for all $i, j, 1 \leqslant j<i \leqslant n$. Tere $p_{1}=1$ ( $\left.\bmod 4\right)$ can be chosen arbitrarily and if $p_{1} \ldots, p_{n}$ have already been chosen in such a way that $p_{i} \equiv 1$. $(\bmod 1), i=1, \ldots, m$, the g.c.d. $\left(p_{i}+p_{j}, p_{1} \ldots p_{m}\right)=1$ for $1 \leqslant j<i \leqslant m$ and $p_{i} \ldots 1$ (mod $\left.p_{j}\right)$, then we pick ap a prime $p_{n+1}$ such that $p_{m+1}$ En (mod $\left.4 p_{j}\right), j=1, \ldots$, wh. Then it is easy to chock that $p_{1}, \ldots, p_{m+1}$ have the roquired proporty (of. [18]).

Now, aceording to Iormma 4.1, thero oxists a real algebraic extonsion 7 of the rationals suld thati

$$
g(h)=\{\mathbf{l},-\mathbf{1}\} \times\left\{\mathbf{1}, \boldsymbol{p}_{\mathfrak{x}}\right\} \times \ldots \times\left\{\mathbf{1}, \boldsymbol{p}_{n-1}\right\}
$$

Trero $q=2^{n}$ and $q_{2}=2^{n-1}$, since every $p_{i}$ is a sum of two squares. Since $p_{i}+p_{j}$ is comrim with $p_{1} \ldots p_{n-1}$, it is a square in $k$, that is, for every pair of indices $i, j$ we havo $\left\langle 1,-p_{i}\right\rangle \approx p_{j}$. Also $\left\langle 1,-p_{i}\right\rangle \approx-1$, j.e. the forms $\langle 1,-p\rangle$ ropresent nil tho basis elements of $g(7)$, hence ench of them is miverwh. Multiplying these universal forms acoording to the 
rule introduced in the proof of Lemma 1.8, we obtain $2^{n-1}$ universal classes. But $u_{2}<2^{n}$ (since $\langle 1,1\rangle$ is not universal), hence $u_{2}=2^{n-1}$ and the statement is proved.

We remarle here that the same method gives also an example of a field $k$ with $q=q_{2}=u_{2}=2^{n}$. We choose $k$ to be non-real with $g(k)=\left\{\mathbf{1}, \boldsymbol{p}_{1}\right\} \times \ldots \times\left\{\mathbf{1}, \boldsymbol{p}_{n}\right\}$. Then as above all the forms $\left\langle 1,-p_{i}\right\rangle=\left\langle 1, p_{i}\right\rangle$ are universal and they generate $2^{n}$ universal forms. Hence we have a field satisfying 2.4.I.

The cases 2.4.II and 2.5.II will be left without examples. I do not know at the moment if the cases actually do occur $(2)$.

In most of the following examples we shall use the fields of formal power series. We state here without proof a lemma giving some known properties of quadratic forms over those fields.

LEMna 4.2. Let $k$ be a field of characteristic not 2 and $K=k((t))$ be the field of formal power series over $k$. Then the following statements hold true.

(4.2.1) $g(K)=g(k) \times\{\mathbf{1}, \boldsymbol{t}\}$, in particular, if $q(k)$ is finite, then $q(\pi)$ $=2 q(k)$.

$(4.2,2)$ If $a, b \in T_{i}^{*}$ and $(a, b)$ is not the hyperbolic plane, then it represents over $K$ onty those square alasses of $g(K)$ which are of the form $c K^{* 2}$, where $e \epsilon k^{*}$ is represented by $(a, b)$ over $k$.

(4.2.3) If $a, b \in k^{*}$, then $(a, b t)$ represents only 2 elements of $g(K)$ over $K$. that is, $\boldsymbol{a}$ and $\boldsymbol{b} t)$.

(4.2.4) The hyperbolic plane is the onty universal class over $\mathbb{K}$, that is, $u_{\mathrm{q}}(K)=1$.

(4.2.5) If $t \mathrm{~s}$ is non-real, then so is $K$ and $s(K)=s(k)$.

The cases 2.4.III and 2.4.IV. For any integer $n \geqslant 3$ and any $m, 1 \leqslant m \leqslant n$, there exists a field $K$ such that $q=q_{2}=2^{n}, u_{2}=1$, every anisotropic binary form represents at most $2^{m}$ elements of $g(K)$ and there is an anisotropic binary form representing exactly $2^{m}$ elements of $g(K)$. For take any field $k$ such that $q(k)=2^{m}, s\left(l_{i}\right)=1$ and every binary form over $k$ is universal. Put $K=k\left(\left(t_{1}\right)\right) \ldots\left(\left(t_{n-m}\right)\right)$ and apply Lemma 4.2 to eheck that $K$ has the required properties. The cases $n=3, m=2$ and $n=3, m=1$ comprise 2.4.III and 2.4.IV, respectively.

The case 2.4.V. For any integer $n \geqslant 3$ there exists a non-real field $K$ such that $q=2^{n}, q_{2}=4$ and $s=4$. Fere the field $K=Q_{2}\left(\left(t_{1}\right)\right) \ldots\left(\left(t_{n-3}\right)\right)$, where $Q_{2}$ denotes the field of 2-adic numbers, satisfies the requirements

(2) Added in proof. I can only prove the following. If there exists a real field satisfying 2.5.II, then a suitable quadratic extension of th is a non-real fiold
satisfying 2.4.II. (for $n=3$ we have $K=Q_{2}$ which falls under $2.4 . \mathrm{V}$ ). It would be desirable to find out whether or not the second possibility (i.e. $s=2$ and $u_{2}=2$ ) appearing in 2.4.V actually oceurs.

The case 2.4.VI. F'or any integer $n \geqslant 3$ there exists a non-real field $K$ such, that $q=2^{n}, q_{2}=4, s=2$ and $u_{2}=1$ (put $n=3$ to obtain 2.4.VI). Let 7 be an algobraic extension of $\boldsymbol{Q}(\sqrt{-2})$ with $g(k)=\{\mathbf{1},-\mathbf{1}\} \times\{\mathbf{1}, \boldsymbol{a}\}$; where $a^{2}=-2$. There $s=2$ and overy binary form is universal (see [17], j. 35), in particular, $q_{2}\left(k_{k}\right)=4$. Put $K=k_{2}\left(\left(t_{1}\right)\right) \ldots\left(\left(t_{n-2}\right)\right)$ and apply Jiemmai 4.2 to oldtain the result

The case 2.4.VII. Fror any integer $n \geqslant 2$ there exists a non-real fietd Wr such that $q:=2^{n}, q_{2}=2, u_{2}=1$. and $s=2(n=3$ gives 2.4.VII). Indeed, let $p$ be a prime number congruent to $3(\bmod 4)$ and put $K$ $=I_{p}\left(\left(t_{1}\right)\right) \ldots\left(\left(t_{n-1}\right)\right)$ or $Q_{p}\left(\left(t_{1}\right)\right) \ldots\left(\left(t_{n-2}\right)\right)$ where $F_{p}$ denotes a prime field of characteristic $p$ and $\boldsymbol{Q}_{p}$ is the field of $p$-adic numbers. Well known behavioun of binary forms over $F_{p}$ and $\boldsymbol{Q}_{p}$. and Lemma 4.2 yield the result.

Now we shall discuss the four remainig cases of Theorem 2.5. First we quote some results of Gross and Fischer concerning the behaviour of the group $g(k)$ under quadratic extensions of $k$. Let $\pi=k(\sqrt{a})$ be a. quadratic extension of the field $k(\operatorname{char} k \neq 2)$. Define $i: g(k) \rightarrow g(K)$ by putting $i\left(b k^{* 2}\right)=b K^{* 2}$ and $N: g(K) \rightarrow g(k)$ by $N\left(\alpha K^{* 2}\right)=N_{K / k^{\prime}}(\alpha) k^{* 2}$. These are group homomorphisms and the sequence

$$
1 \rightarrow\left\{k^{* 2}, a l^{* 2}\right\} \rightarrow g(k) \stackrel{i}{\rightarrow} g(K) \stackrel{N}{\rightarrow} g(k)
$$

is exract (ef. [1], p. 298). From this we get exsily the following result.

Inimun 4.3. Let $k$ be a field with char $k \neq 2$ and $g(k)=\{\mathbf{1}, \boldsymbol{a}\} \times h$, $K=k(\sqrt{a})$. Assume further that $D(1,-a)=\prod_{i \in T}\left\{\mathbf{1}, \boldsymbol{b}_{i}\right\}$ and $c_{i} \in K$ are chosen to satisfy $N_{K / h}\left(o_{i}\right)=b_{i}, i \in T$. Then

$$
g(K)=h \times \prod_{i \in T}\left\{\mathbf{1}, \boldsymbol{c}_{i}\right\}
$$

(here $h$ is meant to be the subgroup of $g(K)$ with the same coset representatives as in $g(k i))$.

Tho case 2.5.trr. Let $k$ bo an real algebraic extension of the rationals such that $g(h)=\{\mathbf{1}, \ldots \mathbf{1}\} \times\{\mathbf{1}, \mathbf{2}\}$ (Lemma 4.1). We consider $\mathbb{K}=k(\sqrt{2})$ and prove that $K$ satisfies 2.5. TrI. The form $(1,-2)$ is universal over he hence $D(1, \ldots 2)=\{\mathbf{1},-\mathbf{1}\} \times\{\mathbf{1}, \mathbf{2}\}$.

Wo have $N_{K / t}(1+\sqrt{2})=-1$ and $N_{K / / 6}(2+\sqrt{2})=2$, hence, by Lemma $4.3,-1,1+\sqrt{2}, 2+\sqrt{2}$ are the representatives of a basis for the groun $g(K)$. Observe that $K$ is a real field and $q=8$. Moreover, $2+\sqrt{2}$ is a stum of two squares,

$$
2+\sqrt{2}=(1+2 \sqrt{2})^{2}+\left(\frac{1}{2} \sqrt{2}\right)^{2}
$$


while $1+\sqrt{2}$ is not, since its conjugate is negative in the ordering of $K$ induced by that of $\boldsymbol{R}$. It follows that $D_{K}(1,1)=\left\{K^{* 2},(2+\sqrt{2}) K^{* 2}\right\}$, i.e. $q_{2}=2$. Further, $u_{2} \leqslant q_{2}=2$ (since the nniversality of $(1, a)$ implies that $-a$ is a sum of two squares) and we prove $u_{2}=2$ by establishing that $(1,-(2+\sqrt{2}))$ is universal. In fact, the equality above implies that $(1,-(2+\sqrt{2})) \approx-1$, and we have also $1-(2+\sqrt{2})=(-1)(1+\sqrt{2})$, that is $(1,-(2+\sqrt{2})) \approx-(1+\sqrt{2})$. Hence the form represents all the basis elements of $g(K)$ and so is universal. Summing up: $K$ is real $q=8, q_{2}$ $=u_{2}=2$, that is, $K$ falls under 2.5.MI.

The case 2.5.IV. For any integer $n \geqslant 3$ there exists a real field $K$ such that $q=2^{n}, q_{2}=2$ and $u_{2}=1$. Talie $k$ as in the preceding example and put $K=k\left(\left(t_{1}\right)\right) \ldots\left(\left(t_{n-2}\right)\right)$. Lemma 4.2 gives the result. When $n=3$ we obtain a field satisfying 2.5.IV.

The case 2.5.V. If $k$ falls under 2.5.V, then, by Proposition 1.22 (ii), the number $r$ of orderings is $\leqslant 3$. On the other hand, the field is pythagorean, so $r \geqslant 3$, by Corollary 1.13. Hence $r=3$ and the field satisfies SAP ([2], Corollary 5.7). Conversely, if $q(k)=8, k$ is pythagorean and satisfies SAP, then $r=3$ and there must be an anisotropic binary form representing more than 2 elements of $g(k)$, since otherwise, by Proposition $1.22(\mathrm{i}), k$ is superpythagorean, i.e. $r=4$.

Hence 2.5.V characterizes pythagorean fields with $q=8$ satisfying SAP. The existence of such a field for any $q$ has been proved by timan and Lam ([2], p. 1187).

The cas e 2.5.VI. By Proposition 1.22(i), the field is superpythagorean. Clearly, for any $n \geqslant 1$ there exists a superpythagorean field with square class number $q=2^{n}$, for example $\boldsymbol{R}\left(\left(t_{1}\right)\right) \ldots\left(\left(t_{n-1}\right)\right)$, as follows directly from Lemma 4.2 .

Remark. The above examples of fields provide at the same time examples for the Classification Theorem 3.2 for Witt groups of fields with $q \leqslant 8$. However, here the number of eases where the existence of fields is not known, increases to 4. In fact, the case 2.4.II splits into two cases $3.2(4.3)$ and $3.2(4.4)$, the case $2.4 . \mathrm{V}$ also splits into two cases one of which (3.2(4.7)) is not covered with any example, and finally, the case 2.5.II goes into $3.2(5.2)$.

\section{References}

[1] C. M. Cordes, The Witt group and the equivalence of fields with respect to quadratic forms, J. Algebra 26 (1973), pp. 400-421.

[2] R. Elm ain and T. Y. Lam, Quadratio forms over formally real fields and pythagorean fields, Amer. J, Math, 94 (1972), pp. 1155-1194.

[3] - - Quadratic form6 and the u-invariant. II, Invent. Math. 21 (1973), pp. 125-
[4] H. Gross and H. R. Fis chex, Non real fields $k$ and infinite dimensional k-vectorspaces, Math. Ann. 159 (1965), pp. 285-308.

[5] I. Kaplansky, Quadratio forms, J. Math. Soc. Japan 5 (1953), pp. 200-207.

[6] - Tröhlioh's local quadratio forms, J. Rieine Angew. Math. 239/240 (1969), pp. 74-77.

[7] - Linear Algebra and Geometry - A second course, Boston 1969.

[8] S. Lang, Algebra, Roading 1971.

[9] F. Lorenz, Quadratiselse Formen über Körpern, Lecture Notes in Mathematice No. 130, Berlin 1970.

[10] O. T. O'Moara, Introduotion to Quadratio Forms, Berlin 1963.

[11] A. Plister, Quadratisohe Formen in beliebigen Körpern, Invent. Math. 1 (1960), pp. 116-132.

[12] W. Scharlau, Quadratische Iformen und Galois-Oohomologie, Invent. Math. $4(1967)$, pp. 238-264.

[13] - Quadratio forms, Queen's Papers on Pure and Applied Mathematics No. 22, Kingstion, Ont., 1969.

[14.] A. Stadok, Grothendieoto groups of quadratio forms over formally real fields, Uniw. Sląski w Katowicach - Prace Mat. 5 (1974), pp. 4.1-47.

[15] L. Szczopanik, Quaternion algebras and binary quadratic forms, Uniw. Sląski w Katowioach - Prace Mat. 6 (1975).

[16] T. Szole, On direct decompositions of abelian groups, J. Luondon Math. Soc. 28 (1953), pp, 24.7-250.

[17] K. SzymiozeK, Grothendiech groups of quadratic forms and Gequivalence of fields, Proe. Cambridgo Philos. Soc. 73 (1973), pp. 29-36. Corrigendum, ibid. $74(1973)$, p. 199.

[18] - Universal binary quadratio forms, Jniw. \$l凤̨ski w Katowicach - Prace Mat. $5(1974)$, pp. 4.9-57.

[19] E. Witit, Theorie der quadratisehen Formen in beliebigen Körpern, J. Reine Angew. Math. 176 (1937), pp. 31-44.

\section{INSTITUTW OF MATHEMATITCS}

STLBSTAY UNTVERSTTY

Katowice

Received on S.4.1974

(561)
AOTA ARTMTMMTICA XXVIIT (1975) 